\title{
Mycoplasma pneumoniae Infection in Children
}

\author{
Gunjan Baweja ${ }^{1}$, Rajdeep Singh ${ }^{2}$
}

\begin{abstract}
Mycoplasma pneumoniae is an important cause of community-acquired pneumonia in school-aged children and adults and the incidence is also increasing in other age groups. It has a varied presentation and apart from respiratory system involvement, it also involves many other systems of the body. In children, central nervous system involvement is very common followed by dermatological, hematological, and other systems. Extrapulmonary manifestations can even cause long-term sequel. Since this organism does not have a cell wall, the diagnosis is always difficult and a strong possibility has to be kept in mind so that the treatment can be started early. The choice of antibiotics is limited, as betalactam antibiotics (which act on the cell wall) do not act on it. In this article, we have reviewed the up-to-date knowledge of $M$. pneumoniae infection in children.
\end{abstract}

Keywords: Atypical pneumonia, Community-acquired pneumonia, Extrapulmonary manifestations, Mycoplasma pneumoniae.

Pediatric Infectious Disease (2021): 10.5005/jp-journals-10081-1243

\section{History and Introduction}

As early in 1898, Nocard and Roux first isolated an agent that was assumed to be the cause of cattle pneumonia and was named as "microbe de la peripneumonie." ${ }^{1-6}$ Microorganisms from other sources were also found to have properties similar to this pleuropneumonia organism (PPO) of cattle, which was known as pleuropneumonia-like organisms (PPLOs), but their true nature was not known. ${ }^{4-6}$ Many PPLO were later proven to be the cause of pneumonia and arthritis in several lower animals. ${ }^{4,7,8}$ In 1930s in the United States, some patients were diagnosed with pneumonia but there symptoms were found to be different from that of bacterial pneumonia or influenza-like viruses. For many years, this organism was known as filterable virus that could pass through the Seitz filter and was recognized as psittacosis-like or a new virus. Later in 1944, Monroe Eaton used embryonated chicken eggs to cultivate an agent thought to be the cause of human primary atypical pneumonia (PAP), commonly known as "walking pneumonia." ${ }^{9}$ It is known as walking pneumonia because of its benign nature specially early in the course of disease.

\section{Epidemiology}

Pneumonia is the most common presentation of Mycoplasma. Its incidence is more common in school-aged children and adults but it causes infection in all age groups. The geographic presentation of Mycoplasma infection is also varied, involving almost all the countries. ${ }^{10}$ The exact incidence of Mycoplasma pneumoniae infections remains underdiagnosed because of its presumed benign nature, lack of diagnostic tests with good sensitivity and specificity, and its clinical presentations mimic with other infections also. The incidence is found to be higher in patients who are suffering from asthma and other chronic lung conditions. There have been recent outbreaks in children and adults according to the Centers of Disease Control and Prevention (CDC). ${ }^{11}$ Pneumonia due to $M$. pneumoniae increases with age ( $2 \%$ in children $<2$ years, $5 \%$ in age group 2-4 years, $16 \%$ in the age group 5-9 years, and $23 \%$ in the age group 10-17 years). ${ }^{12}$
1Department of Pediatrics, JP Medical Centre, Chandigarh, India
${ }^{2}$ Department of Medicine, JP Medical Centre, Chandigarh, India

Corresponding Author: Gunjan Baweja, Department of Pediatrics, JP Medical Centre, Chandigarh, India, Phone: +91 9872086223, e-mail: gunjandr@hotmail.com

How to cite this article: Baweja G, Singh R. Mycoplasma pneumoniae Infection in Children. Pediatr Inf Dis 2021;3(3):95-98.

Source of support: Nil

Conflict of interest: None

\section{Pathogenesis}

There are five genera (Mycoplasma, Ureaplasma, Acholeplasma, Anaeroplasma, Asteroloplasma) in the class Mollicutes and the term Mycoplasma is used for any of these organisms. ${ }^{2}$ Mycoplasma pneumoniae is the fastidious organism that lack the cell wall, though it can be grown under both anaerobic and aerobic conditions and also can be isolated on the media supplemented with serum. Mycoplasma pneumoniae is divided into two genetic groups, subtype 1 and subtype 2. Mycoplasma pneumoniae infection is likely a consequence of the interplay between the two subtypes, each emerging after the other induces transient herd immunity. ${ }^{13}$ The most important intrinsic virulence factors include cytoadherence and mobility. The main anchor proteins $\mathrm{P} 1$ and $\mathrm{P} 30$ are attached to the polar terminal organelle of the pathogen, which help in adherence of the organism. The infection is associated with elevated mRNA levels of cytokines, including interleukin (IL)-8, tumor necrosis factor- $a$, and IL-1 $\beta$, which lead to the recruitment of the inflammatory cell. ${ }^{10,14}$

The mechanism of infection include:

- Direct infection with the organism at the site of inflammation and activation of local cytokines.

- Indirect infection by modulating the immune system that may involve cross-reactivity between bacterial and human cells. These include cold agglutinins to I-antigen of human red blood cells. ${ }^{15}$

- Vasculitis and/or thrombosis. ${ }^{16}$ 


\section{Clinical Manifestations}

The symptoms of $M$. pneumoniae infection in children can be varied. It can be just an asymptomatic carrier or it can cause mild respiratory symptoms like cold and cough or can involve lower respiratory infections like pneumonia, asthma-like symptoms, and leading to more serious manifestations involving the other systems of the body. The clinical profile of M. pneumoniae infections is discussed further in Table 1.

Table 1: Pulmonary and extrapulmonary manifestations of $M$. pneumoniae infection $^{1-3}$

\begin{tabular}{ll}
\hline Organ involvement & Manifestations \\
\hline Pulmonary & 1. Asthma \\
2. Chronic obstructive pulmonary disease & (COPD) exacerbation \\
3. Tracheobronchitis & 4. Pneumonia: lobar and multilobar infiltrates \\
5. Diffuse alveolar hemorrhage
\end{tabular}

Respiratory system involvement is the most common manifestation of $M$. pneumoniae infection in children. Most of them have mild upper respiratory symptoms, which are usually self-limiting. Its onset is gradual starting with cough, fever, headache, malaise, sore throat, leading to breathing difficulty and gradually involving other systems of the body. Cough is the important manifestation; it can be dry or productive because of tracheobronchitis and can even persist for days to weeks. Community-acquired pneumonia is the common presentation in school-aged children. The person remains in the carrier state for a time much longer than symptoms and it does not change by the antibiotic therapy.

Neurological manifestations are the next common in children. The incubation period for the development of central nervous system (CNS) symptoms after the respiratory symptoms manifest varies from 2 to 23 days (mean 10 days), but in about $5 \%$ of patients there may not be any preceding respiratory symptoms. Encephalitis within 5 days of the prodromal symptoms may be because of the direct invasion of the organism in the brain. Encephalitis after 7 days of prodromal symptoms may be because of the autoimmune response and is more common than the direct infection. Other CNS manifestations are described in the Table 1.

Dermatological manifestations may range from simple maculopapular rash to bullous papular purpuric gloves and socks syndrome, urticaria rash, and in some children more severe manifestations like Steven Johnson syndrome (SJS). Some studies have proved that the cause of SJS may be the direct invasion of bacteria in skin. Other skin manifestations like urticaria, anaphylactoid purpura, and erythema multiforme are most likely immunologically mediated.

Hematological system involvement in the form of hemolysis can occur due to the cold agglutinin response due to appearance of IgM antibodies against I antigen on the erythrocyte membrane. This may be clinically insignificant in normal patients but can be severe in patients with underlying diseases like sickle cell anemia.

The other important extrapulmonary manifestations are discussed in Table 1. In children with community-acquired pneumonia and multisystem involvement, M. pneumoniae should always be considered.

\section{Diagnosis}

Since M. pneumoniae is without cell wall, diagnosing it is always a challenge.

In community-acquired pneumonia, X-ray chest is always done for confirmation, though in $5 \%$ of patients with M. pneumoniae pneumonia, X-ray chest may be normal. Common patterns in chest $X$-ray of $M$. pneumoniae patients are the following:

- Peribronchial and perivascular interstitial infiltrates (49\%)

- Airspace consolidation (38\%)

- Reticulonodular opacification (8\%)

- Nodular or mass-like opacification (5\%)

Other findings include pleural effusion, cavitary disease, and hilar lymphadenopathy. ${ }^{1}$

Total leucocyte count and inflammatory markers like C-reactive protein (CRP) and the erythrocyte sedimentation rate (ESR) may be elevated but they do not differentiate it from other causes of pneumonia and are not helpful. In patients with CNS involvement, cerebrospinal fluid (CSF) shows lymphocytic pleocytosis, elevated protein, and normal glucose. ${ }^{2}$ 
There is no single confirmatory test for M. pneumoniae infection.

Cultures on special media (SP4 agar media) of the sputum sample may show classical mulberry colonies but it requires the incubation period of 7-21 days and is not done routinely in most of the laboratories.

Antigen detection can be done by antigen capturing EIA (AgElA), which may detect $10^{4}$ colony-forming units $/ \mathrm{mL}$. The disadvantage of using this is that the ability to detect the antigen may vary with time of sampling and it has been largely replaced by molecular testing. The best method of diagnosis is the combination of the nucleic acid amplification test (NAAT) including the PCRbased assay and the serology. ${ }^{2,10}$ The PCR of the nasopharyngeal and throat swab for M. pneumoniae DNA has a sensitivity and specificity of 80 and $>97 \%$. The PCR is more reliable in the acutely ill patients and the infection becomes undetectable by PCR once the antibiotic therapy is started.

Fourfold increase in antibiotic titers remains the gold standard for diagnosis (IgM antibody titers rise earlier than IgG). Both PCR and serology may not be concordant in elderly and infants due to an insufficient immunological response toward the infection.

Cross-reacting antibodies against red blood cells called cold agglutinin titer may be raised in patients with $M$. pneumoniae infection by 2 nd week of illness and resolves by $6-8$ weeks. In a child with pneumonia, titers more than 1:64 can be due to $M$. pneumoniae, but has low specificity. There is also cross-reactivity with EpsteinBarr virus (EBV). ${ }^{17}$ Cold agglutinins may be elevated in $50-60 \%$ of Mycoplasma pneumoniae pneumonia (MPP) patients but can also be present in EBV, cytomegalovirus, or Klebsiella infections.

Other modern diagnostic techniques that lack widespread validity include nanorod array-surface-enhanced Raman spectroscopy (NA-SERS) and matrix-assisted laser desorption ionization-time of flight mass spectrometry (MALDI-TOF MS). ${ }^{10}$

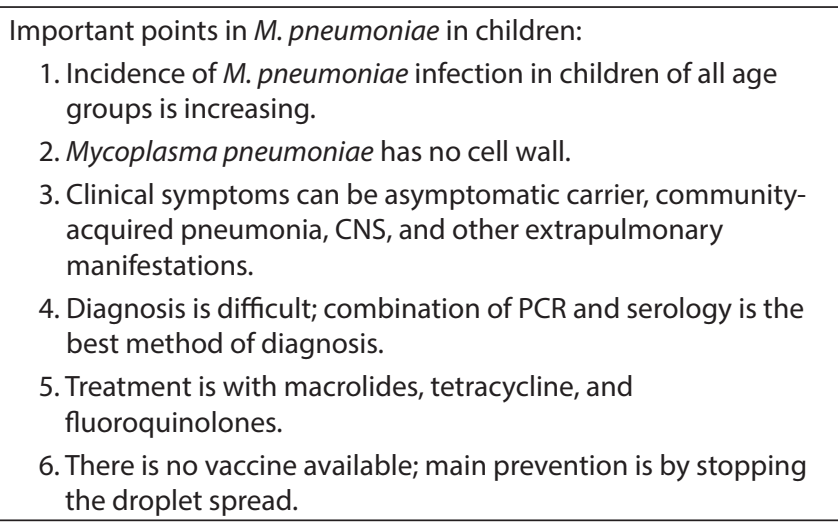

\section{Treatment}

Mycoplasma pneumoniae infection resolves on its own in 7-10 days but treatment is often necessary. Since $M$. pneumoniae is without the cell wall, the choice of antibiotics is also limited as beta-lactam antibiotics that act on the cell walls do not act on it and hence the antibiotics that act on the ribosome remain the choice of treatment. Since the diagnosis is difficult early in the disease, empiric antibiotics are usually started. The choice of antibiotics include macrolides, ketolides, streptogramins, and tetracyclines. Macrolides are bacteriostatic and have immunomodulatory action. Macrolides and ketolides bind to specific nucleotides of the 23S rRNA in the 50S bacterial ribosomal subunit, blocking protein synthesis by causing premature dissociation of peptidyl-tRNA from the ribosome. ${ }^{10,18}$ Azithromycin has long half-life and has better tolerance and hence remains the drug of choice. ${ }^{2}$

Dose of azithromycin is $10 \mathrm{mg} / \mathrm{kg}$ on day 1 orally or intravenously (max $500 \mathrm{mg}$ ), followed by $5 \mathrm{mg} / \mathrm{kg}$ on day 2 to day 5 (max $250 \mathrm{mg}$ ).

Dose of clarithromycin is $15 \mathrm{mg} / \mathrm{kg}$ per day in two divided doses for 10 days ( $\max 1 \mathrm{~g}$ ).

The other drugs like tetracycline can be used in children more than 8 years of age. The dose of doxycycline is $2-4 \mathrm{mg} / \mathrm{kg}$ orally or intravenously in one to two divided doses for 10 days (max 200 mg per day).

There has been widespread resistant to macrolides called macrolide-resistant M. pneumoniae (MRMP), which was first seen in Japan and later seen in many other countries. In a study done in 8 hospitals of United States during 2015-2018, macrolide resistance was seen in $7.5 \%$ of total patients. ${ }^{19}$ As an alternative, fluoroquinolones have been used as second-line treatment in immunocompetent children and an alternative in immunocompromised patients specially if they have macrolide exposure before.

Dose of levofloxacin is for children $>5$ years of age, $10 \mathrm{mg} / \mathrm{kg}$ per dose (max $750 \mathrm{mg}$ per day) once a day orally or intravenously for 10 days.

For children 6 month to 5 years, the dose is $8-10 \mathrm{mg} / \mathrm{kg}$ per dose in two divided doses orally or intravenously (max $750 \mathrm{mg}$ per day) for 10 days.

Extrapulmonary manifestations may need adjunctive treatment. Steroids, diuretics, and immunotherapy (intravenous immunoglobulin and plasmapheresis) can be used in patients with severe life-threatening conditions with CNS, hematological involvement, and dermatological conditions like SJS. ${ }^{3}$

\section{Prevention}

In hospitalized patients, precautions should be taken to prevent the spread through respiratory droplets. Mycoplasma pneumoniae remains contagious for a long time even after the antibiotic therapy, which should be explained to the patients. There have been many vaccine trials for $M$. pneumoniae but none has been successful and there is no vaccine at present for the prevention. There is no role of giving prophylactic antibiotics to the contacts, though can be considered in household contact with high-risk patients like sickle cell anemia. ${ }^{2,3}$

\section{References}

1. Bajantri B, Venkatram S, Diaz-Fuentes G. Mycoplasma pneumoniae: a potentially severe infection. J Clin Med Res 2018;10(7):535-544. DOl: 10.14740/jocmr3421w.

2. Vallego JG, et al., Mycoplasma pneumoniae infection in children. Uptodate January 2020.

3. Mejias A, Ramila O. Mycoplasma infections. ed. Kliegman RobertM. Nelson Text book of Pediatrics. 20th ed., Philadelphia: Elsevier; 2016. pp. 1487-1490.

4. Hayflick L, Chanock RM. The mycoplasma (PPLO) species of man. Bacteriol Rev 1965;29(2):185-221. DOI: 10.1128/MMBR.29.2.185221.1965.

5. Hayflick L, Stanbridge E. Isolation and identification of mycoplasma from human clinical material. Ann New York Acad Sci 1967;143(1):5-6. DOI: 10.1111/j.1749-6632.1967.tb27636.x.

6. Hayflick L. The Mycoplasmatales and the L-phase of bacteria. New York: New Holland Publishing Company; 1969. 
7. Marmion BP. Eaton agent-science and scientific acceptance: a historical commentary. Rev Infect Dis 1990;12(2):338-353. DOI: 10.1093/clinids/12.2.338.

8. Saraya T. The history of mycoplasma pneumoniae pneumonia. Front Microbiol 2016;7:364. DOI: 10.3389/fmicb.2016.00364.

9. Eaton MD, Meiklejohn G, van Herick W. Studies on the etiology of primary atypical pneumonia. A filterable agent transmissible to cotton rats, hamsters, and chick embryos. J Exp Med 1944;79(6): 649-668. DOI: 10.1084/jem.79.6.649.

10. Waites KB, Xiao L, Liu Y, et al. Mycoplasma pneumoniae from respiratory tract and beyond. Clin Microbiol Rev 2017;30(3):747-809. DOI: 10.1128/CMR.00114-16.

11. Diaz MH, Benitez AJ, Winchell JM. Investigations of mycoplasma pneumoniae infections in United States: trends in molecular typing and macrolide resistance from 2006 to 2013. J Clin Microbiol 2015;53(1):124-130. DOI: 10.1128/JCM.02597-14.

12. Jain S, Williams DJ, Arnold SR, et al. Community acquired pneumonia requiring hospitalization among U.S. children. New Engl J Med 2015(9):372-835. DOI: 10.1056/NEJMoa1405870.

13. Dumke R, Catrein I, Herrmann R, et al. Preference, adaptation and survival of Mycoplasma pneumoniae subtypes in animal models. Int J Med Microbiol 2004;294(2-3):149-155. DOI: 10.1016/ j.ijmm.2004.06.020.

14. Yang J, Hooper WC, Phillips DJ, et al. Cytokines in Mycoplasma pneumoniae infections. Cytokine Growth Factor Rev 2004;15(23):157-168. DOI: 10.1016/j.cytogfr.2004.01.001.

15. Janney FA, Lee LT, Howe C. Cold hemagglutinin crossreactivity with Mycoplasma pneumoniae. Infect Immun 1978;22(1):29-33. DOI: 10.1128/IAI.22.1.29-33.1978.

16. Narita M. Pathogenesis of extrapulmonary manifestations of Mycoplasma pneumoniae infection with special reference to pneumonia. J Infect Chemothe 2010;16(3):162-169. DOI: 10.1007/ s10156-010-0044-x.

17. Waites KB, Crabb DM, Duffy LB. In vitro activities of ABT-773 and other antimicrobials against human mycoplasmas. Antimicrob Agents Chemothe 2003;47(1):39-42. DOI: 10.1128/aac.47.1.39-42.2003.

18. Waites KB, Brabb DM, Duffy LB. Comparative in vitro susceptibilities of human mycoplasmas and ureaplasmas to a new investigational ketotide CEM - 101. Antimicrobial Agents Chemothe 2009;53(5): 139-2141. DOI: 10.1128/AAC.00090-09.

19. Kutty PK, Jain S, Taylor TH, et al. Mycoplasma pneumoniae among children hospitalized with community acquired pneumonia. Clin Infect Disease 2019;68(1):5. DOI: 10.1093/cid/ciy419. 